\title{
Towards a Cyber-physical Systems Resilience Approach based on Artificial Emotions and Multi-agent Systems
}

\author{
Eskandar Kouicem, Clément Raïevsky and Michel Occello \\ Univ. Grenoble Alpes, Grenoble INP, LCIS, 26000 Valence, France \\ \{firstname.lastname $\} @$ lcis.grenoble-inp.fr
}

\begin{abstract}
Keywords: Artificial Emotions, Multi-agent Systems, Cyber-physical Systems, Resilience, Distributed Systems.
Abstract: $\quad$ The concept of resilience is popular and studied in different domains like human and social sciences (psychology, psychiatry, sociology etc.) and recently in cognitive science, biology, ecology and computer science. The objective of this article is to present our research that aims to incorporate knowledge from human and social sciences in computer science to solve cyber-physical systems resilience problems. For us humans, emotions are considered as an important process in responding to unanticipated events, for that, emotions are important for our resilience. Our work aims to inspire from emotional processes to create an agent model that increases the resilience of cyber-physical systems. This agent model will integrate individual and collective processes. In addition, one of our principal hypotheses in our research is that the multi-agent paradigm is suitable to integrate emotion-like processes into cyber-physical systems.
\end{abstract}

\section{INTRODUCTION}

As humans, our emotions increase our ability to adapt to unknown situations as individuals and as groups (Frijda, 1986; Lazarus, 1991). So human emotion is a relevant source of inspiration for solving distributed systems resilience problems that we consider as the ability to recognize, adapt, and handle unanticipated perturbations (Woods, 2012). For us, a cyberphysical system (CPS) is a system in which its mechanisms are controlled or monitored by computer-based algorithms, composed of several subsystems and it has at least a part of its subsystems in direct interaction with the physical world. Depending on their characteristics, which are the interaction with the physical environment and the open system, cyber-physical systems can have some problems such as: incorrect collected data, communication losses, limited resources, adding or removing components to/from the CPS during the execution, and the unpredictability of the encountered situations. We therefore aim to use knowledge from psychology of emotion and sociology to improve cyber-physical systems' resilience, especially taking into consideration their distributed aspect. To this end, we adopt the multi-agent paradigm which intrinsically and historically support using social and cognitive metaphors in the design and implementation of distributed complex systems (Ferber and Weiss, 1999). This approach will allow us to in- tegrate cognitive science and psychology knowledge about resilience (resilience to stress (Lazarus, 1993), collective resilience etc.) and cognitive functions of emotion in agents' decision-making mechanisms and in the self-organizing processes of the agents groups.

This paper starts by defining resilience in different domains and presents a classification of existing resilience approaches and their problems. After that, we present our analysis of the relevance of emotion-related cognitive processes in our resilience and how to draw from these cognitive processes to improve resilience-related artificial processes in cyberphysical systems. Relationships between resilience properties and characteristics of emotion and multiagent systems are then proposed. Finally, we conclude this paper by the description of the perspective of this work.

\section{RESILIENCE}

Resilience is studied by researchers in a variety of disciplines including psychology, sociology, and recently cognitive science, ecology and computer science.

The term resilience is used differently by different communities. Ruault et al. (2011) explain that, in ecology, resilience is the ability of an ecosystem or species to recover its normal behaviour after experi- 
encing traumas. They also state that in psychology, resilience is the ability of a person or a group to develop well, to continue to project into the future, despite destabilizing events, difficult living conditions and sometimes severe traumas. At the level of the individual, traumas destroy the psyche, at the level of the group, traumas destroy the existing bonds between the members of the group.

Generally, in systems engineering, resilience is a rapid recovery from a degraded system state. The networks community defines it as the combination of reliability (reliability, security, performance) and tolerance (survivability, disturbance tolerance and traffic tolerance). The computer science community has defined resilience as the persistence of supplying services and the availability of features (Trivedi et al., 2009).

According to Woods (2012), in resilience engineering, "resilience concerns the ability to recognize and adapt to handle unanticipated perturbations that call into question the model of competence, and demand a shift of processes, strategies and coordination".

There is often a confusion in the uses of the terms robustness and resilience. To disambiguate the two, we chose the definitions of (Linkov et al., 2013b):

- Robustness is the extent to which a system is able to withstand an unexpected internal or external event or change without degrading system performance.
- Resilience refers to the system's ability to recover or regenerate its performance after an unexpected impact produces a degradation of its performance (Linkov et al., 2013b).

\subsection{Resilience in Cyber-physical Systems}

To position our approach relative to the cyberphysical systems (CPSs) domain, we based our study on the work of Lee et al. (2015) who defined the 5C architecture presented in Figure 1. This architecture specifies how a CPS is built, from the initial data acquisition to the creation of final system.

The integration of all the 5 levels in a CPS is rarely achieved. Generally, a CPS has two main functional components:

1. advanced connectivity that provides real-time data acquisition from the physical world and information about cyberspace, and

2. intelligent data management, data analysis and computing capabilities that build cyberspace.

However, this requirement is very abstract and not specific enough for CPS implementation in general (Lee et al., 2015).

Since our aim is to give CPS the ability to selfconfigure and self-adapt in order to improve their resilience, it appears that our contribution will be a part

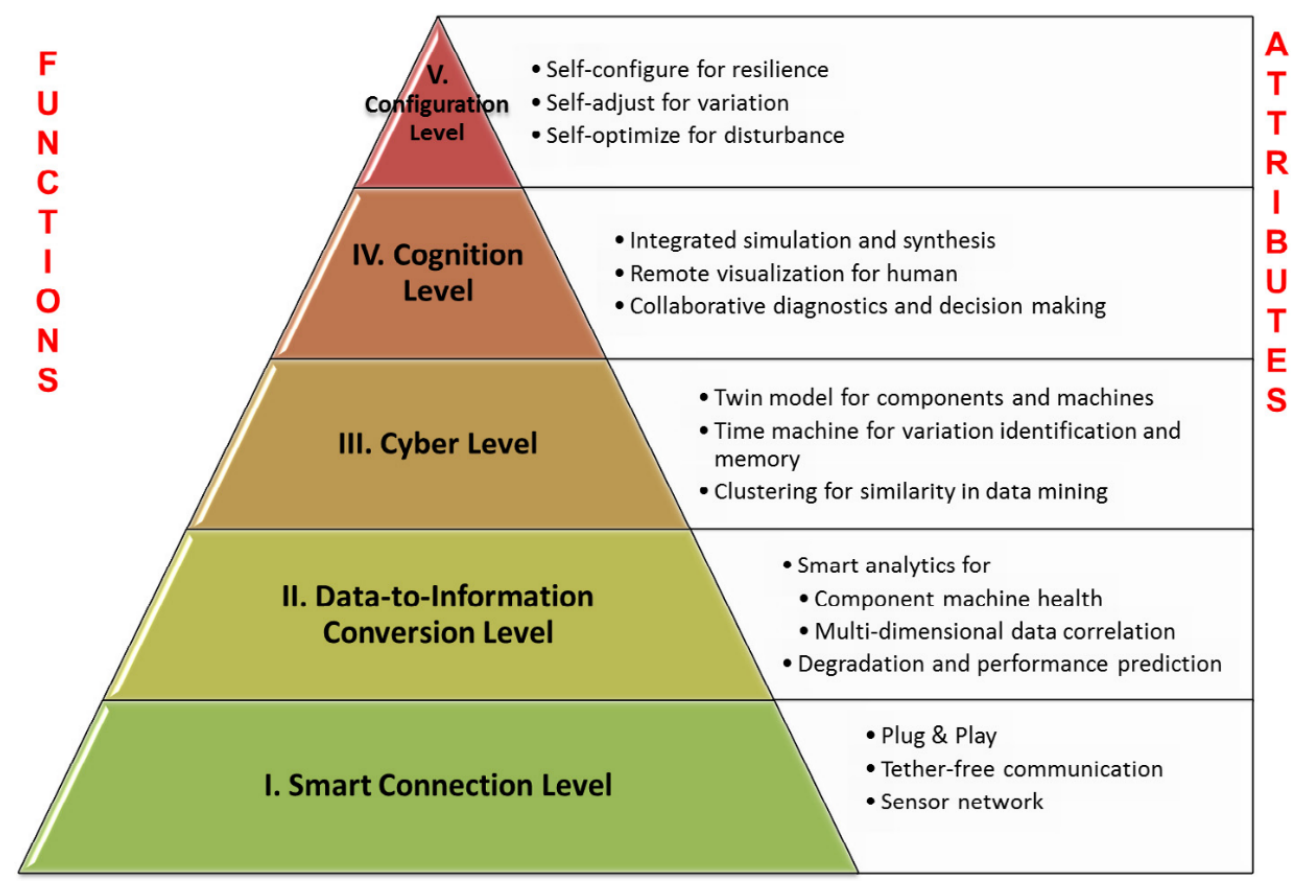

Figure 1: 5C architecture for the implementation of a cyber-physical system (Lee et al., 2015). 
of the upmost layer of the 5C architecture: "V. Configuration Level".

\subsection{Existing Resilience Approaches}

Existing resilience approaches are divided into two categories: qualitative and quantitative (see Figure 2) (Hosseini et al., 2016).

- The qualitative category: it includes methods that assess the resilience of a system without any numerical descriptor, it also contains two subcategories:

- Conceptual Frameworks: offer best practices for analyzing system's resilience by guiding resilient systems principles and characteristics.

- Semi-quantitative Indices: provide expertise on different qualitative aspects of resilience. These approaches are developed using a set of questions designed to assess different resiliencebased characteristics (e.g., redundancy, resourcefulness).

- Quantitative methods with two sub-categories:

- General Resilience Measures: determine resilience by comparing the system performance before and after the disturbance without focusing on the specific system's characteristics. There are deterministic and stochastic measures, each one of them is used to describe the static and dynamic system's behaviour.

- Structural-based Approaches: examine how the structure of a system affects its resilience by observing the system's behaviour and modelling or simulating the characteristics of the system.

Another classification of existing resilience approaches into two other main categories: metricbased and model-based approaches (see Figure 3) (Linkov and Kott, 2019).

- Metric-based Approaches: use individual component properties or system functions measures (metrics) to assess overall the system's performance. Resilience metrics are defined as a measurable properties of the system that quantify the system's objectives achievement degree.

- Model-based Approaches: use system configuration modeling and scenario analysis to determine overall the system's performance. These approaches use mathematical or physical concepts to represent the real world (environment) and define the resilience. Modelling the system's resilience requires the knowledge of a system's critical functions, tasks, objectives, temporal patterns, thresholds, memory, and adaptation.

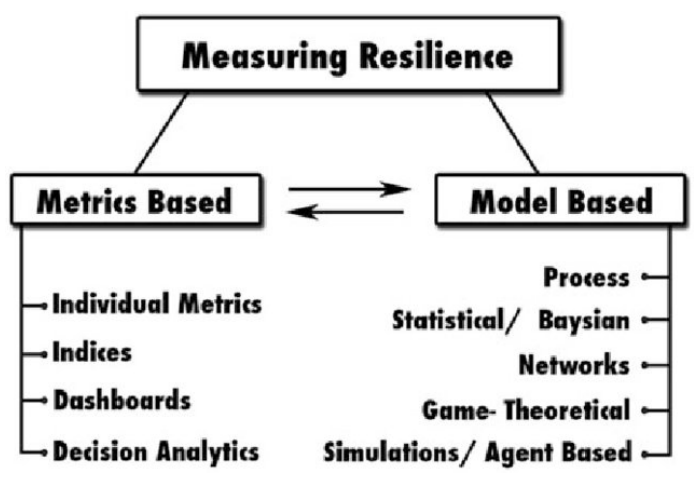

Figure 3: Metric-based and model-based approaches for resilience assessment (Linkov and Kott, 2019).

According to Hosseini et al. (2016), the main approaches addressing the issue of resilience are either centralized or based on redundancy. In contrast we aim for taking the distributed nature of CPS into account at the core of the resilience-related processes and to avoid redundancy because it is not always practically feasible in systems including physical components.

\section{PROPOSED SOLUTION}

As we can see in Figure 3, agent-based approach and multi-agent systems are considered as model-based approaches to address resilience.

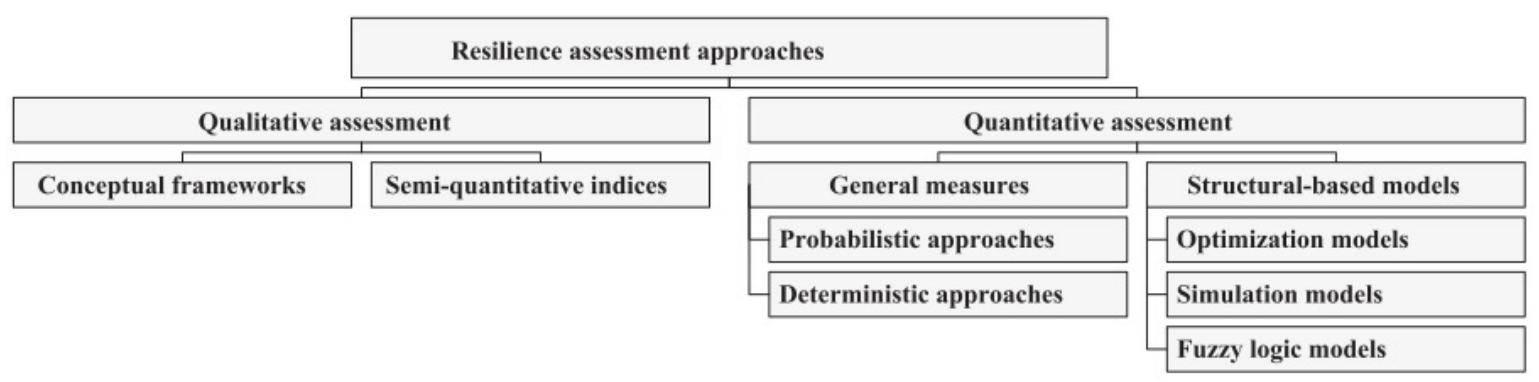

Figure 2: Classification scheme of resilience assessment methodologies (Hosseini et al., 2016). 
Our work aims to increase resilience in a cyberphysical system by providing its subsystems with a form of autonomy of decision that allows them to detect abnormal situations and adapt their behaviour to these situations. These mechanisms are inspired by human emotions and allow the entire cyber-physical system to detect abnormal situations, and then, adapt its behaviour to these situations using artificial processes inspired by social metaphors (Kouicem et al., 2019). To do so, our approach utilizes the multi-agent paradigm (Ferber and Weiss, 1999).

\subsection{Resilience Profile}

Figure 4 presents our adaptation of the resilience profile proposed by Linkov and Kott (2019). This profile represents the different resilience-related behaviours triggered by the occurrence of an advert event.

We added a detection behaviour phase which is active before the event and crucial to CPSs since it is responsible for the triggering of absorption and recovery behaviour. Once the event is detected and the absorption is active, the system begins the recovery process, potentially taking into account information about the event gathered by the detection behaviour.

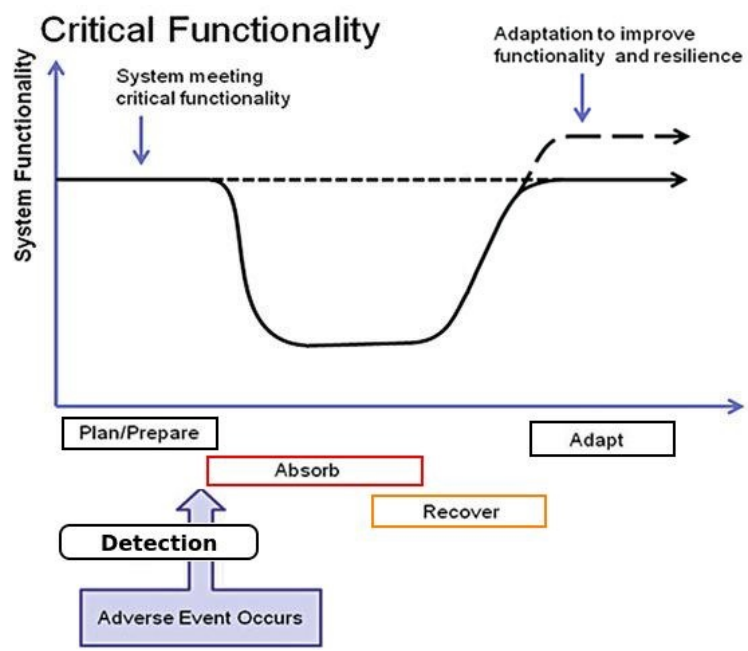

Figure 4: The extended resilience profile (based on (Linkov and Kott, 2019)).

As we see, it is possible that the system will improve its functionality and resilience during the adaptation phase. This increase is justified by the addition of the potential gain in the detection and recovery capacity acquired during the adaptation phase to the system's functionality.

\subsection{Multi-agent Approach for CPS Resilience}

As we have said, in our proposed solution we use a social metaphor for cyber-physical systems. So we have chosen the multi-agent approach because it suits best our needs, adapted to the control of distributed systems and addressing certain aspects of resilience.

Multi-agent systems offer us a decentralized solution to solve the "single point of failure" problem inherent in centralized solutions. In this approach, each agent is autonomous and it has its own role, there is no global control, the data and the decisions are decentralized (Ferber and Weiss, 1999). Through these characteristics a multi-agent system can maintain its functioning in case of communication loss, decreases the volume of transmitted data, scaling up and avoids redundancy/replication of many software and hardware components of the cyberphysical system using the autonomy of its agents. Moreover, adopting the multi-agent paradigm and self-organization (Di Marzo Serugendo et al., 2006) breaks with traditional resilience approaches by using a social metaphor for cyber-physical systems. Adding to this, this paradigm allows us to use cognitive science and psychology to reproduce the cognitive functions of emotions (Ivanović et al., 2015), both in the individual's decision-making process and in the group's self-organization process.

There is some work on resilience in cyberphysical systems using a multi-agent approach (Januário et al., 2018, 2019), that solves the problem of resilience enhancement in CPSs and WSAN (wireless sensor and actuator network) using hierarchical multi-agent systems. They propose frameworks based on a multi-agent approach implemented in a distributed middleware. These frameworks aim to improve the resilience of networked control and supervision systems. Our proposal is largely distinct because we aim to use artificial processes inspired by human emotions and social metaphors to improve cyber-physical systems resilience.

\subsection{Use of Artificial Emotions for CPS Resilience}

According to Frijda (1986) emotional phenomena are: "non-instrumental behaviours and non-instrumental features of behaviour, physiological changes, and evaluative subject-related experiences, as evoked by external or mental events, and primarily by the significance of such events." 
In computer science, artificial emotions are a set of pre-programmed or non-scheduled processes running within a machine, facilitating decision-making and enabling the system to adapt to the environment. The artificial emotion is the fruit of the program's input-output as well as its own internal activity, and is often the object of a collaboration with a cognitive structure, by means of which the system deals with the problems introduced by its environment. In addition, it is part of a programming logic more or less explicit, but still in the field of computable (Mahboub, 2011; Kouicem et al., 2019).

The main existing work uses artificial emotions for human-machine interaction and the detection and expression of natural emotions (Calvo et al., 2015). Other work uses artificial emotions to make virtual agents behaviours more realistic (Saunier and Jones, 2014; Adam et al., 2010). More specifically, in the field of cyber-physical systems, the main work concerns the emotion recognition (Calero et al., 2018) and emotional robots (Breazeal, 2003). We noticed that this work falls within the scope of affective computing (Picard, 2000).

We note that our work differs from these research themes, it does not deal with the interaction with humans or the simulation of natural emotions, but rather by inspiring from their functions (Raïevsky and Michaud, 2009). In existing work on artificial emotions, emotions are elicited by an analysis of symbolic events related to the objectives of the system or require a large amount of knowledge of the system designer about the situations that the system may encounter. These initial hypotheses of existing work limit the resilience of the resulting systems. Furthermore, these characteristics are difficult to apply to CPSs because:

1. They are embedded in the physical world and can not trigger emotions from symbolic data about the situation and,

2. the distributed and opened nature of CPSs prevents their designers from anticipating all possible situations.

In order for cyber-physical systems to benefit from the capacities provided by emotions, without compromising their resilience, we propose to identify generic mechanisms, not specific to the task of the system, to initiate the artificial equivalent of an emotional episode. Otherwise, artificial emotions will mainly be used to detect abnormal situations and update social organizations in response to these abnormal situations. These functions are directly related to the "Plan/Prepare","Absorb" and "Adapt" phases of the resilience profile depicted in Figure 4.
The existing work that links resilience with emotions is mainly in psychology and sociology (Masten et al., 1990; Lazarus, 1991, 1993; Tugade and Fredrickson, 2004; Norris et al., 2008). We note that our idea of creating an emotion-based approach to increase cyber-physical systems resilience remains original.

\subsection{Resilience Matrix}

In resilience profile (see Figure 4), we can see the four stages of the event management cycle that a system needs to maintain to be resilient according to the National Academy of Sciences (NAS) (National Academy of Sciences, 2012; Linkov et al., 2013b,a) which are:

1. Plan/Prepare: lay the foundation to ensure that services and assets remain available in case of abnormal situations.

2. Absorb: maintain the availability of the most critical functions and services while delaying or isolating the disruption.

3. Recover: restore all asset functions and service availability to their pre-event (before the abnormal situation) functionality.

4. Adapt: use knowledge of critical events to modify communication protocols, system configuration, learning process or other aspects to increase resilience.

Linkov et al. (Linkov et al., 2013a,b) combined those four phases and four domains (physical, information, cognitive and social) to create a generic matrix of resilience metrics. In our case, we combined the four phases of resilience with artificial emotions (cognitive) and multi-agent systems (social) for obtaining our resilience matrix (see Table 1). This mapping helped us to identify the aspects of artificial emotions and multi-agent systems (cells of the matrix) that we will integrate in our agent model to increase cyber-physical systems resilience. In the matrix, we used the detection phase as a combination of "Plan/Prepare" and "Absorb" phases because it fully covers the planning phase and trigger the absorption phase (see Figure 4).

\subsection{Scenario (An Example)}

We consider a cyber-physical system composed of several sensors and actuators as a multi-agent system, each subsystem (sensor or actuator) of the CPS is an agent. A sensor agent perceives data from the environment with its sensor module. An actuator 
agent acts on the environment with its actuator module. Agents who share the same goals belong to the same organization, whether they are sensors or actuators. The agents of an organization communicate with each other, so the actuator agents share with the other agents their operating status and the sensor agents provide information about the environment. So, if an actuator agent needs some information, it asks the sensor agents of its organization to perceive data for it, if these ones can't get it, they can ask the sensor agents of the other organizations. Each sensor agent stores the recently perceived data in a time series database in its own memory, then the old time series will be shared in the memories of the actuators of its same organization. Basically, the agents have a predefined tolerance thresholds to the perceived data stocked in both sensor and actuator agents.

Here is a reaction scenario of the agents based on emotional processes in case of an anomaly in the data that a sensor agent perceives from the environment:

- Detection: the detection is always active, before any critical event until the beginning of the absorption phase. Once a sensor agent has a doubt about the data that it perceives by getting out-ofdomain values unusual sequences or long repetition of similar data, the planning phase begins.

- Plan/Prepare: the sensor agent increases the sampling frequency and compares the data with a larger time series and communicates the situation to the other agents of his organization to confirm whether it is a true abnormal or just a false positive.

- Absorb: if the situation is confirmed as abnormal, the agents reduce the tolerance thresholds and communicate this situation to other organizations of the system.
- Recover: the system's agents continue to operate ignoring the data of this sensor agent and take into consideration the system's data history in the same conditions.

- Adapt: the agents use the knowledge of this situation by modifying their communication protocol intra-organization and/or extra-organization or updating the organizations architectures to improve its resilience.

\section{CONCLUSIONS AND FUTURE WORK}

In this paper, we presented our work about utilizing the multi-agent paradigm to integrate knowledge from psychology of emotion to improve cyber-physical systems resilience. We presented our study of the definition of resilience in relevant research domains and presented our proposition and our choices regarding inspiration from human emotion as well as the choice of the multi-agent paradigm.

Our goal is to create an agent model based on the resilience matrix that has been defined. This agent model integrates individual and decentralized processes dedicated to the detection of abnormal situations inspired by the cognitive processes triggering emotional episodes in humans. The agent model will also include mechanisms for adapting individual behaviour in relation with the detected situations in order to increase the resilience of the cyber-physical system. Individual detection and adaptation trigger collective processes in the system, these processes use the information built by the individual mechanisms. The individual behaviour adaptation impacts on the organization of the agents group that controls

Table 1: The resilience matrix.

\begin{tabular}{|c|c|c|c|}
\hline & $\begin{array}{l}\text { Detection phase: Plan/Prepare and } \\
\text { Absorb }\end{array}$ & Recover & Adapt \\
\hline $\begin{array}{l}\text { Artificial } \\
\text { emotions }\end{array}$ & $\begin{array}{l}\text {-Using an incremental process for } \\
\text { anomaly detection. } \\
\text {-Anticipating existing scenarios. } \\
\text {-Increase the sampling frequency. } \\
\text {-Increasing in the readiness for action. } \\
\text {-Decrease of tolerance thresholds. }\end{array}$ & -Rapid recovery of nominal behaviour. & $\begin{array}{l}\text {-Review the response and decision-making } \\
\text { processes. } \\
\text {-Adaptation of the response behaviour of } \\
\text { the situation. }\end{array}$ \\
\hline $\begin{array}{l}\text { Multi-agent } \\
\text { systems }\end{array}$ & $\begin{array}{l}\text {-Collective detection process. } \\
\text {-Propagation of detected situations. } \\
\text {-Cooperation for negotiate a detected } \\
\text { situation to decide if it is a real or a } \\
\text { fake abnormal situation. }\end{array}$ & $\begin{array}{l}\text {-Establish a collective decision making } \\
\text { techniques or aids to select recovery } \\
\text { options. }\end{array}$ & $\begin{array}{l}\text {-Sharing the responses (adaptations). } \\
\text {-A self-organization of the group of agents } \\
\text { carrying out the system's control. } \\
\text {-Improve decision-making and allow } \\
\text { the system to adapt to the environment. }\end{array}$ \\
\hline
\end{tabular}


the cyber-physical system, which will trigger selforganization for adapting the collective behaviour to the abnormal situation.

The proposed agent model and multi-agent system will be tested by simulation (Jamont and Occello, 2013), and also in real cyber-physical systems in order to quantify experimentally the resilience improvement they bring.

\section{ACKNOWLEDGEMENTS}

This Ph.D project has received funding from the Trust research chair of the Grenoble-INP Foundation and the Auvergne-Rhône-Alpes region.

\section{REFERENCES}

Adam, C., Canal, R., Gaudou, B., Vinh, H. T., Taillandier, P., et al. (2010). Simulation of the emotion dynamics in a group of agents in an evacuation situation. In International Conference on Principles and Practice of Multi-Agent Systems, pages 604-619. Springer.

Breazeal, C. (2003). Emotion and sociable humanoid robots. International journal of human-computer studies, 59(1-2):119-155.

Calero, J. A. M., Marino, R., Lanza-Gutierrez, J. M., Riesgo, T., Garcia-Valderas, M., and Lopez-Ongil, C. (2018). Embedded emotion recognition within cyberphysical systems using physiological signals. In 2018 Conference on Design of Circuits and Integrated Systems (DCIS), pages 1-6. IEEE.

Calvo, R. A., D'Mello, S., Gratch, J. M., and Kappas, A. (2015). The Oxford handbook of affective computing. Oxford University Press, USA.

Di Marzo Serugendo, G., Gleizes, M.-P., and Karageorgos, A. (2006). Self-organisation and emergence in multiagent systems: An overview. Informatica, 30(1):4554.

Ferber, J. and Weiss, G. (1999). Multi-agent systems: an introduction to distributed artificial intelligence, volume 1. Addison-Wesley Reading.

Frijda, N. H. (1986). The emotions. Cambridge University Press.

Hosseini, S., Barker, K., and Ramirez-Marquez, J. E. (2016). A review of definitions and measures of system resilience. Reliability Engineering \& System Safety, 145:47-61.

Ivanović, M., Budimac, Z., Radovanović, M., Kurbalija, V., Dai, W., Bădică, C., Colhon, M., Ninković, S., and Mitrović, D. (2015). Emotional agents-state of the art and applications. Computer Science and Information Systems, 12(4):1121-1148.

Jamont, J.-P. and Occello, M. (2013). Using mash in the context of the design of embedded multiagent system. In International Conference on Practical Applications of Agents and Multi-Agent Systems, pages 283-286. Springer.

Januário, F., Cardoso, A., and Gil, P. (2018). Multi-agent framework for resilience enhancement over a wsan. In 2018 15th International Conference on Electrical Engineering/Electronics, Computer, Telecommunications and Information Technology (ECTI-CON), pages 110-113. IEEE.

Januário, F., Cardoso, A., and Gil, P. (2019). A distributed multi-agent framework for resilience enhancement in cyber-physical systems. IEEE Access, 7:31342_ 31357.

Kouicem, E., Raïevsky, C., and Occello, M. (2019). Artificial Emotions for Distributed Cyber-physical Systems Resilience. In Proceedings of the Cyber-Physical Systems PhD Workshop 2019, pages 84-95.

Lazarus, R. S. (1991). Emotion and adaptation. Oxford University Press on Demand.

Lazarus, R. S. (1993). From psychological stress to the emotions: A history of changing outlooks. Annual review of psychology, 44(1):1-22.

Lee, J., Bagheri, B., and Kao, H.-A. (2015). A cyberphysical systems architecture for industry 4.0-based manufacturing systems. Manufacturing Letters, 3:1823.

Linkov, I., Eisenberg, D. A., Bates, M. E., Chang, D., Convertino, M., Allen, J. H., Flynn, S. E., and Seager, T. P. (2013a). Measurable resilience for actionable policy. Environmental Science \& Technology, 47(18):1010810110.

Linkov, I., Eisenberg, D. A., Plourde, K., Seager, T. P., Allen, J., and Kott, A. (2013b). Resilience metrics for cyber systems. Environment Systems and Decisions, 33(4):471-476.

Linkov, I. and Kott, A. (2019). Fundamental concepts of cyber resilience: Introduction and overview. In $C y$ ber resilience of systems and networks, pages 1-25. Springer.

Mahboub, K. (2011). Modélisation des processus émotionnel dans la prise de décision.(Emotional processes modelling in decision making). $\mathrm{PhD}$ thesis, University of Le Havre, France.

Masten, A. S., Best, K. M., and Garmezy, N. (1990). Resilience and development: Contributions from the study of children who overcome adversity. Development and psychopathology, 2(4):425-444.

National Academy of Sciences, N. (2012). Disaster resilience: A national imperative. Washington, DC: The National Academies Press.

Norris, F. H., Stevens, S. P., Pfefferbaum, B., Wyche, K. F., and Pfefferbaum, R. L. (2008). Community resilience as a metaphor, theory, set of capacities, and strategy for disaster readiness. American journal of community psychology, 41(1-2):127-150.

Picard, R. W. (2000). Affective computing. MIT press.

Raïevsky, C. and Michaud, F. (2009). Emotion generation based on a mismatch theory of emotions for situated agents. In Handbook of Research on Synthetic Emotions and Sociable Robotics: New Applications in Af- 
fective Computing and Artificial Intelligence, pages 247-266. IGI Global.

Ruault, J.-R., Luzeaux, D., Colas, C., and Sarron, J.C. (2011). Résilience des systèmes sociotechniques application à l'ingénierie système. Génie logiciel, 96:40-52.

Saunier, J. and Jones, H. (2014). Mixed agent/social dynamics for emotion computation. In Proceedings of the 2014 international conference on Autonomous agents and multi-agent systems, pages 645-652. International Foundation for Autonomous Agents and Multiagent Systems.

Trivedi, K. S., Kim, D. S., and Ghosh, R. (2009). Resilience in computer systems and networks. In Proceedings of the 2009 International Conference on ComputerAided Design, pages 74-77. ACM.

Tugade, M. M. and Fredrickson, B. L. (2004). Resilient individuals use positive emotions to bounce back from negative emotional experiences. Journal of personality and social psychology, 86(2):320.

Woods, D. D. (2012). Essential characteristics of resilience. In Resilience engineering, pages 33-46. CRC Press. 